\title{
Imatinib-induced apoptosis: a possible link to topoisomerase enzyme inhibition
}

\author{
Y. Baran* PhD, S. Zencir† MSc, Z. Cakir* BSc, E. Ozturkł BSc and Z. Topcu§ PhD \\ *Department of Molecular Biology and Genetics, Faculty of Sciences, Izmir Institute of Technology, Izmir, \\ $\dagger$ Department of Biochemistry, Faculty of Sciences, Ege University, Izmir, †Department of Biology, Faculty \\ of Sciences, Ege University, Izmir, and §Department of Pharmaceutical Biotechnology, Faculty of \\ Pharmacy, Ege University, Izmir, Turkey
}

\section{SUMMARY}

What is known and Objective: Imatinib is a specific BCR/ABL inhibitor, commonly used for the treatment of chronic myeloid leukaemia (CML), a hematological malignancy resulting from a chromosomal translocation that generates the BCR/ABL fusion protein. Recent studies showed that the imatinib has cytotoxic and apoptotic effects on many BCR/ABL-negative cancers. Numerous compounds with cytotoxic potential exert their functions by interfering with the DNA topoisomerase. In this study, we examined the effects of imatinib on tumour cell-killing in relation to DNA topoisomerase enzyme inhibition.

Methods: We determined the cytotoxicity by cell proliferation assay (XTT; tetrazolium hydroxide), using the human K562 CML cells, and loss of mitochondrial membrane potential by monitoring the changes in caspase- 3 enzyme activity. Type I and II topoisomerase activities were measured by supercoiled plasmid relaxation and minicircle DNA decatenation assays respectively.

Results and Discussion: Imatinib-induced apoptosis and inhibited cell proliferation in a dosedependent manner. We also found that the imatinib was effective in both type I and type II topoisomerase reactions to a varying degree between $94 \%$ and $7 \%$ for the concentration range of $1 \mathrm{~mm}-0.02 \mathrm{~mm}$ in a dose-dependent manner.

Received 01 April 2010, Accepted 17 October 2010

Correspondence: Z. Topcu PhD, Department of Pharmaceutical Biotechnology, Faculty of Pharmacy, Ege University, 35100 Izmir, Turkey. Tel.: +90 232388 4000/ext 1931; fax: +90 232388 5258; e-mail: zeki.topcu@ege.edu.tr
What is new and Conclusion: Our results suggest that the inhibition of topoisomerases may be a significant factor in imatinib-induced apoptosis in CML.

Keywords: apoptosis, BCR/ABL, chronic myeloid leukaemia, imatinib, topoisomerase

\section{WHAT IS KNOWN AND OBJECTIVE}

Chronic myeloid leukaemia (CML) is a hematopoietic stem cell disorder, characterized by an increased number of immature white blood cells (1, 2). CML is diagnosed by the presence of $B C R / A B L$ oncogene, resulting from a reciprocal translocation between the long arms of chromosomes 9 and 22, and formation of the diagnostic Philadelphia chromosome $(\mathrm{Ph})$. The $B C R / A B L$ hybrid gene encodes a fusion protein with constitutive tyrosine kinase activity that induces leukaemogenesis (3). Imatinib is the first target-specific anticancer agent for the treatment of cancers including Ph-positive CML and gastrointestinal stromal tumours (4). It specifically recognizes and binds to the ATP binding site of $\mathrm{BCR} / \mathrm{ABL}$ and inhibits its activity (5). Inhibition of $\mathrm{BCR} / \mathrm{ABL}$ activity modulates the expression of several genes involved in the regulation of cell growth, proliferation, apoptosis, cell cycle, cell adhesion and differentiation (6).

Platelet-derived growth factor receptors (PDGFR), colony stimulating factor 1 receptor (CSF1R) and c-KIT are also the targets of imatinib. These molecules are highly expressed in many BCR/ABL-negative cancer types such as dermatofibrosarcoma protuberans (DFSP), melanoma, chordoma and aggressive fibromatosis, and gastrointestinal stromal tumours (GIST). In GIST, imatinib 
blocks the activity of mutant or wild-type c-KIT tyrosine kinase $(7,8)$. We have previously reported that imatinib induces apoptosis by increasing the intracellular concentrations of apoptotic ceramides via generation and accumulation of ceramides by upregulating ceramide synthase genes and downregulating the anti-apoptotic sphingosine kinase-1 gene (9). We have evidence indicating that imatinib induces autophagy and programmed cell death-II, by increasing the expression levels of Atg5, Atg6 and LC-3 genes in a dose-dependent manner (unpublished data). However, the mechanisms of imatinibinduced apoptosis in BCR/ABL-negative cancers are yet to be clarified.

Numerous naturally occurring and synthetic compounds with cytotoxic and anticancer properties interfere with the normal DNA topoisomerase functions (10-16) and DNA-protein interactions (17). Topoisomerases are essential enzymes playing important roles in many genetic processes, including the DNA replication, transcription, recombination and transposition (18). There are two types of topoisomerases; Type I topoisomerases (topo I) make a single-stranded break in a DNA duplex, mediate passage of the intact strand through the break, and then reseal it. Type II topoisomerases, on the other hand, create transient breaks in both the strands of a duplex, pass an intact DNA segment through the break and then reseal the cleavage site $(19,20)$. All known type II topoisomerases require ATP for their catalytic activities in topoisomerization reactions.

In this study, we have determined the cytotoxicity and loss of mitochondrial membrane potential using imatinib. As topoisomerases have been recognized as an effective target for the development of chemotherapeutics, we extended our research to cover the mammalian DNA topoisomerase assays for assessing the biological activities of imatinib.

\section{METHODS}

\section{Cell culture}

Human K562 CML cells were obtained from German Collection of Microorganisms and Cell Cultures and maintained in RPMI1640 medium containing $10 \%$ foetal bovine serum and $1 \%$ streptomycin-penicillin at $5 \% \mathrm{CO}_{2}$ incubator at $37^{\circ} \mathrm{C}$ (NuAire Laboratory Equipment Supply, MN, USA).

\section{XTT Cell proliferation assay}

Cell proliferations were determined by XTT cell proliferation assay (Biological Industries, Kibbutz Beit Haemek, Israel) as described (21). $2 \times 10^{4}$ cells were grown in a 96-well plates containing $200 \mu \mathrm{L}$ growth medium per well in the absence or presence of increasing concentrations of imatinib and incubated at $37^{\circ} \mathrm{C}$ in $5 \% \mathrm{CO}_{2}$ incubator. Following $72 \mathrm{~h}$ incubation, $50 \mu \mathrm{L}$ XTT reagent was added to each well and then incubated for another $4 \mathrm{~h}$. Finally, the absorbance was measured by ELISA reader under $490 \mathrm{~nm}$ wavelength of light (Thermo Electron Corporation Multiskan Spectrum, Vantaa, Finland).

\section{Detection of apoptosis by caspase-3 enzyme activity and mitochondrial membrane potential (MMP)}

Changes in caspase-3 enzyme activity in response to imatinib were measured by caspase- 3 colorimetric assay as described (R\&D Systems, Minneapolis, USA) (21). Briefly, the K562 cells were treated with different concentrations of imatinib and incubated for $72 \mathrm{~h}$. The cells were collected by centrifugation at $800 \mathrm{~g}$ for $10 \mathrm{~min}$. The pellets were lysed on ice for $10 \mathrm{~min}$, centrifuged at $10000 \mathrm{~g}$ for 1 min and supernatants were transferred into a new tube. To determine caspase-3 enzyme activity, $50 \mu \mathrm{L}$ of reaction buffer (5X), $5 \mu \mathrm{L}$ of caspase- 3 colorimetric substrate, and $50 \mu \mathrm{L}$ of the samples were applied to the cell suspensions in 96-well plate and incubated for $2 \mathrm{~h}$ at $37^{\circ} \mathrm{C}$ in $5 \% \mathrm{CO}_{2}$ incubator. Absorbance of the samples was read under $405 \mathrm{~nm}$ wavelength of light via the ELISA (Thermo Electron Corporation Multiskan Spectrum, Vantaa, Finland). Protein concentrations were determined by Bradford assay and caspase- 3 enzyme activity levels were normalized to protein concentrations.

\section{Loss of mitochondrial membrane potential (MMP)}

The mitochondrial membrane potential was detected by JC-1 MMP kit as described by the manufacturer (Cell Technology, CA, USA). K562 cells were treated with imatinib as described above. The pellets were collected by centrifugation at $800 \mathrm{~g}$ for $10 \mathrm{~min}$ and then treated with $500 \mu \mathrm{L}$ of JC-1 dye before their incubation at $37{ }^{\circ} \mathrm{C}$ in $5 \% \mathrm{CO}_{2}$ 
for $15 \mathrm{~min}$. These mixtures were centrifuged at $800 \mathrm{~g}$ for $5 \mathrm{~min}$, and then mixed with the $2 \mathrm{~mL}$ of blank media. After centrifugation at $800 \mathrm{~g}$ for $5 \mathrm{~min}$, the pellets were dissolved in $500 \mu \mathrm{L}$ of blank media and $150 \mu \mathrm{L}$ from each sample was transferred into 96-well plates. The aggregate red form of the dye which is found in inner mitochondria has absorption/emission maxima of 585/590 nm, and the monomeric green form of the dye which was released to the cytoplasm due the loss of the MMP has absorption/emission maxima of 510/ $527 \mathrm{~nm}$. The plate was read at these wavelengths by fluorescence Elisa reader ELISA (Thermo Electron Corporation Multiskan Spectrum, Vantaa, Finland). Finally, green/red (510/585) values were calculated to determine the changes in MMP.

\section{Topoisomerase I supercoiled plasmid relaxation assay}

Supercoiled plasmid relaxation assays were carried out as previously described $(14,22)$. Briefly, $20 \mu \mathrm{L}$ of reaction mixture contained $500 \mathrm{ng}$ of superhelical circular (sc) plasmid DNA, one unit of calf thymus topo I (TAKARA, Otsu-Shiga, Japan) in the presence or absence of the Imatinib in the reaction buffer ( $35 \mathrm{~mm}$ Tris- $\mathrm{HCl}$ (pH 8.0), $72 \mathrm{~mm} \mathrm{KCl,} 5 \mathrm{~mm}$ $\mathrm{MgCl}_{2}, 5 \mathrm{~mm}$ DTT, $5 \mathrm{~mm}$ spermidine and $0 \cdot 1 \%$ bovine serum albumin). Relaxation products were separated on $1 \%$ agarose gels in TBE buffer ( $45 \mathrm{~mm}$ Tris-Borate and $1 \mathrm{~mm}$ EDTA, $\mathrm{pH} 8.0$ ) in a horizontal electrophoresis apparatus $(5 \mathrm{~V} / \mathrm{cm})$ (Thermo EC250, Waltham, MA, USA). Electrophoresis was continued until the bromophenol blue dye had migrated $75 \%$ down the gel and photographed under UV light after staining in ethidium bromide (Etd-Br) solution $(0.5 \mu \mathrm{g} / \mathrm{mL})$. The relationship between the binding of Etd-Br and the amount of fluorescence given by sc and relaxed DNA (rlx DNA) under UV light was carried out as described $(23,24)$. DNA bands were quantified from gel photo images using BioRad Multianalyst (ver. 1·1). Average band intensities were calculated from three independent reactions.

\section{Topoisomerase II decatenation assays}

Decatenation assays were carried out using $0 \cdot 2 \mu \mathrm{g}$ kinetoplast DNA (K-DNA) substrate in a final volume of $20 \mu \mathrm{L}$ in topo II reaction buffer $(50 \mathrm{~mm}$
Tris-Cl, pH 8.0, $120 \mathrm{~mm} \mathrm{KCl}, 10 \mathrm{~mm} \mathrm{MgCl} 2.0 \cdot 5 \mathrm{~mm}$ ATP, $0.5 \mathrm{~mm}$ dithiothreitol) (25). Reactions were terminated with stop buffer (5\% sarkosyl, $0 \cdot 0025 \%$ bromophenol blue, $25 \%$ glycerol) and electrophoretic analyses of K-DNA were performed using standard agarose gel containing $0.5 \mu \mathrm{g}$ Etd-Br $/ \mathrm{mL}$. Resolution of decatenated DNA products were monitored as stated above except the gels were destained in water prior to the photography. Etoposide, a well-known topoisomerase II inhibitor was used as a control.

\section{RESULTS AND DISCUSSION}

\section{Imatinib inhibits proliferation of K562 cells in a dose-dependent manner}

We examined the proliferation rates of K562 cells in response to increasing concentrations of imatinib for $72 \mathrm{~h}$ by XTT assay. The results revealed that there were $9 \%, 14 \%, 22 \%, 27 \%, 35 \%$ and $87 \%$ decreases in cell proliferation in response to $0.5,5$, $50,100,200$ and $600 \mathrm{~nm}$ imatinib respectively, as compared with untreated controls. We could not detect any significant change in the proliferation of K562 cells in response to $0 \cdot 1 \mathrm{~nm}$ imatinib as compared with the untreated controls whereas $600 \mathrm{~nm}$ imatinib showed the highest anti-proliferative effect (Fig. 1). IC50 (drug concentrations that

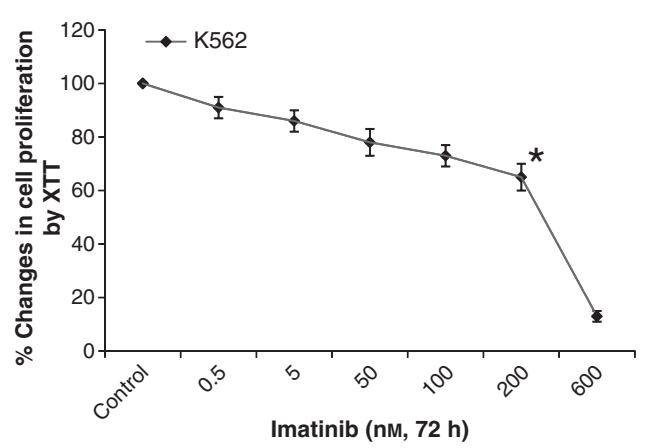

Fig. 1. Effects of imatinib on the growth of K562 cells. The IC50 concentration of imatinib was calculated from cell proliferation plots. The XTT assays were performed using triplicate samples in at least two independent experiments. The error bars represent the SD, and when not seen, they are smaller than the thickness of the lines on the graphs. Statistical significance was determined using ANOVA, and $(P<0.05)$ was considered significant. [Correction made on 12 October 2011, figure 1 has been replaced with the corrected figure.] 
inhibits $50 \%$ of cell proliferation) value of imatinib, calculated from cell proliferation plots was $310 \mathrm{~nm}$ (Fig. 1). [Corrections added on 12 October 2011, after online publication: The sentence 'The results revealed that there were $6 \%, 12 \%, 16 \%, 26 \%, 80 \%$ and $84 \%$ decreases in cell proliferation in response to $0.1,1,10,100,500$ and $1000 \mathrm{~nm}$ imatinib respectively, as compared with untreated controls (Fig. 1)' was corrected to 'The results revealed that there were $9 \%, 14 \%, 22 \%, 27 \%, 35 \%$ and $87 \%$ decreases in cell proliferation in response to $0.5,5$, $50,100,200$ and $600 \mathrm{~nm}$ imatinib respectively, as compared with untreated controls', '500 nm imatinib' was corrected to ' $600 \mathrm{~nm}$ imatinib' and $280 \mathrm{~nm}$ was corrected to $310 \mathrm{~nm}$.]

\section{Imatinib induces apoptosis in a dose-dependent manner}

To examine the apoptotic effects of imatinib on K562 cells, we incubated the cells with four different concentrations of imatinib and examined the changes in caspase-3 enzyme activity and loss of mitochondrial membrane potential. We choose IC50 and lower concentrations of imatinib (50, 150, 250 and $300 \mathrm{~nm}$ ) to assess the strength of apoptotic effects of imatinib. The results revealed that incubation of K562 cells in the presence of 50, 150, 250 and $300 \mathrm{~nm}$ imatinib resulted in 13\%, 37\%, 40\% and $54 \%$ increases in caspase- 3 enzyme activity compared with the untreated control group (Fig. 2), To confirm these results, we examined the changes in MMP and observed that the same doses of imatinib caused 14\%, 28\%, 46\% and 51\% increases in cytoplasmic/monomeric JC-1 relative to the untreated control cells (Fig. 3). The observed differences seen with both caspase-3 enzyme activity and mitochondrial membrane potential were statistically significant $(P>0 \cdot 05)$. [Corrections added on 12 October 2011, after online publication: 'incubated the cells with two different concentrations of imatinib' was corrected to 'incubated the cells with four different concentrations of imatinib', the sentence 'We choose IC50 dose, $280 \mathrm{~nm}$ and $10 \mathrm{~nm}$ to assess the strength of apoptotic effects of imatinib' was corrected to 'We choose IC50 and lower concentrations of imatinib (50, 150, 250 and $300 \mathrm{~nm}$ ) to assess the strength of apoptotic effects of imatinib', the sentence 'The results revealed that incubation of K562 cells in the presence of 10 and

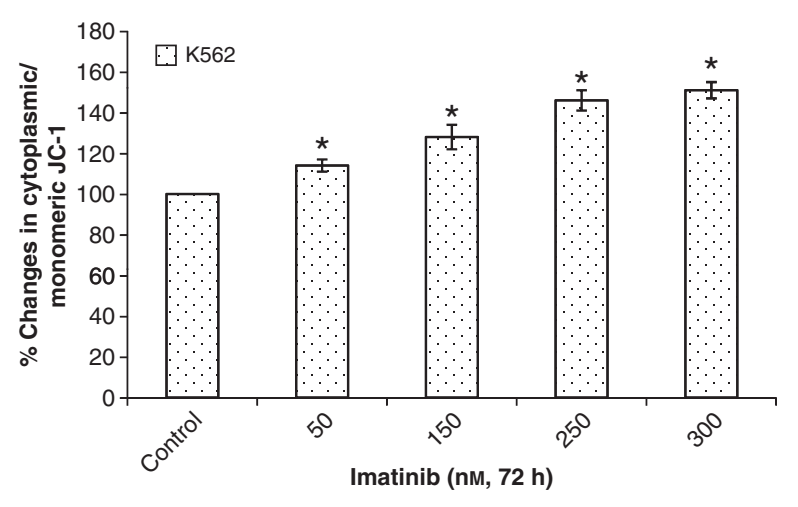

Fig. 2. Percent changes in caspase-3 enzyme activity in imatinib exposed $\mathrm{K} 562$ cells. The results are the means of two independent experiments. The error bars represent the SD. Statistical significance was determined using ANOva, and $(P<0.05)$ was considered significant. [Correction made on 19 January 2011, figure 2 has been replaced with the corrected figure.]

$280 \mathrm{~nm}$ imatinib resulted in $27 \%$ and $58 \%$ increases in caspase- 3 enzyme activity compared with the untreated control group (Fig. 2) was corrected to 'The results revealed that incubation of K562 cells in the presence of 50, 150, 250 and $300 \mathrm{~nm}$ imatinib resulted in $13 \%, 37 \%, 40 \%$ and $54 \%$ increases in caspase-3 enzyme activity compared with the untreated control group (Fig. 2) and ' $31 \%$ and $50 \%$ increases' was corrected to ' $14 \%, 28 \%, 46 \%$ and $51 \%$ increases']

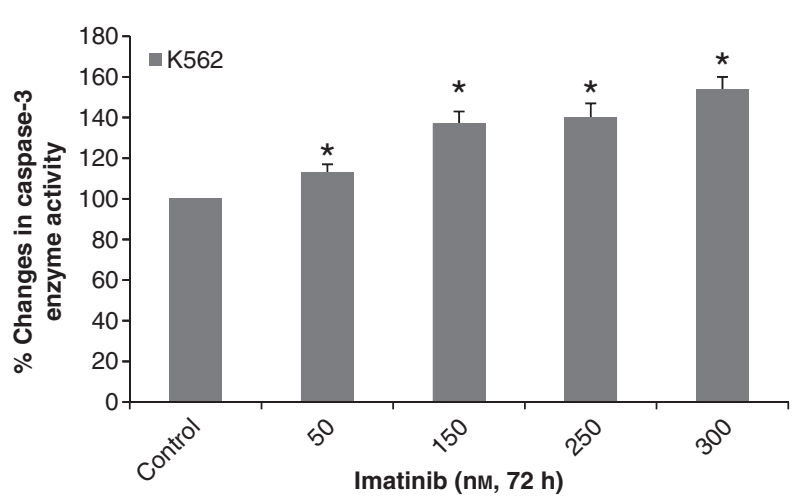

Fig. 3. Percent changes in cytoplasmic/monomeric JC-1 in imatinib treated $\mathrm{K} 562$ cells. The results are the means of two independent experiments. The error bars represent the SD. Statistical significance was determined using ANOvA, and $(P<0.05)$ was considered significant. [Correction made on 12 October 2011, figure 3 has been replaced with the corrected figure.] 
(a)

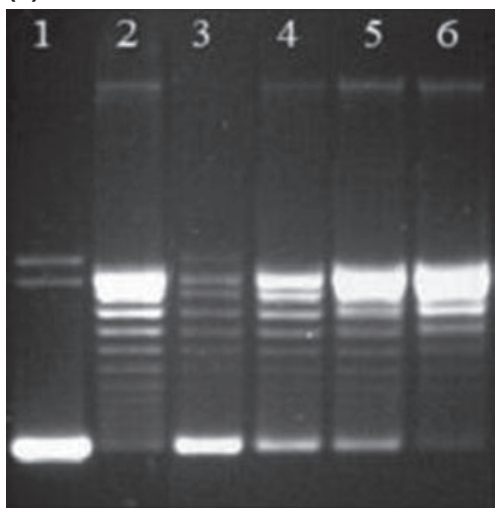

(b)

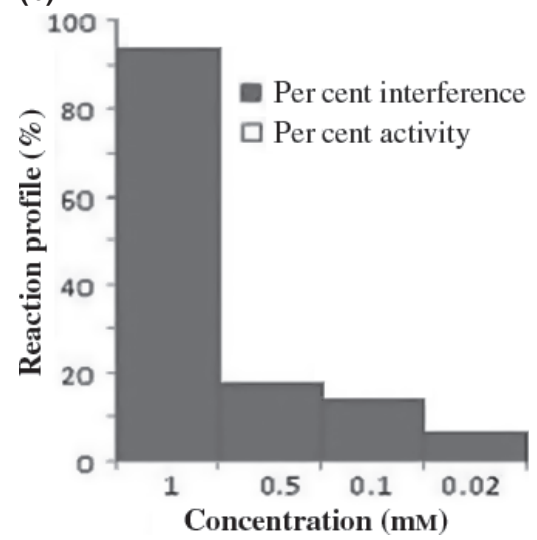

Fig. 4. The effect of imatinib on mammalian DNA topoisomerase I activity. (a) A representative agarose gel photograph of supercoil relaxation in the presence of varying concentrations of imatinib; lane 1, pBR322; lane 2, pBR322 with 1 U of DNA topoisomerase I; lanes 3-6, same as lane 2 plus $1 \mathrm{~mm}, 0.5 \mathrm{~mm}, 0.1 \mathrm{~mm}$ and $0.02 \mathrm{~mm}$ Imatinib respectively. (b) Quantitative assessment of the reaction profile corresponding to samples from lanes 3 to 6 (see 'Materials and methods' for the details).

\section{Imatinib interferes with both type I and type II topoisomerase reactions}

We carried out plasmid DNA nicking and DNA decatenation assays to monitor the effect of imatinib on topoisomerase I and topoisomerase II reactions respectively. Topoisomerase I assay employs supercoiled plasmid DNA (sc DNA) and relies on the ability of the enzyme to convert sc DNA to its relaxed form, which can be separated as discrete bands using gel electrophoresis. An inhibition of relaxation activity due to the presence of a particular inhibitor was monitored in the form of an accumulated faster-migrating sc DNA. On the other hand, topoisomerase II assay was based on the decatenation of K-DNA, minicircles from the insect trypanosome Crithidia fasciculata (25). As K-DNA is an aggregate of interlocked DNA minicircles, resulting high molecular weight would prevent the substrate population from entering the gel, unless the K-DNA is decatenated by the enzyme, topoisomerase II. Inclusion of Etd-Br in the gel enabled us to monitor open-circular monomers and covalently closed circular DNA bands.

A representative assay using various concentrations of imatinib is given in Fig. 4. As seen in Fig. 4A, sc plasmid substrate, pBR322 (Fig. 4A, lane 1 ) is relaxed by topo I (Fig. $4 \mathrm{~A}$, lane 2) in the absence of imatinib. However, the reaction was profoundly affected by $1 \mathrm{~mm}$ imatinib (Fig. $4 \mathrm{~A}$, lane 3). The interference by imatinib gradually decreased upon the dilution to imatinib to $0.5 \mathrm{~mm}$ and $0.1 \mathrm{~mm}$ (Fig. 4A, lanes 4 and 5 respectively). This effect completely disappeared when the concentration of imatinib was lowered to $0.02 \mathrm{~mm}$ (Fig. 4A, lane 6). At this level, only a very faint sc DNA band with an accumulated lower migrating relaxed DNA (rlx DNA) was seen. Densitometric calculations of relative band intensities showed that the interference was $94 \%$ at $1 \mathrm{~mm}$ imatinib concentration and this value gradually decreased

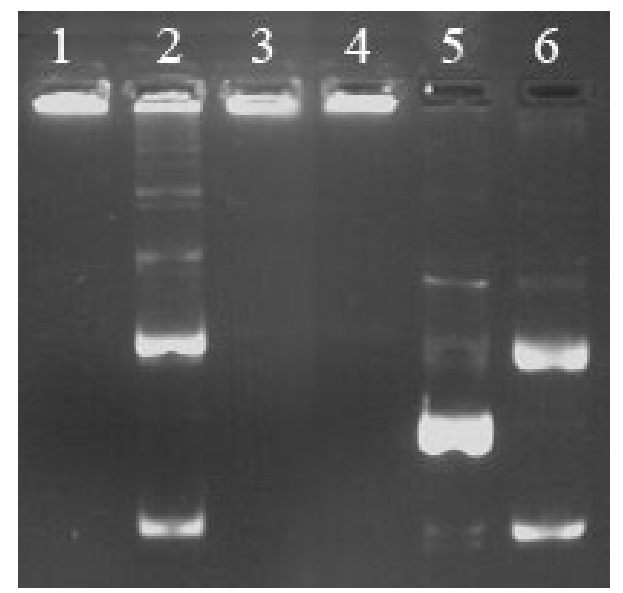

Fig. 5. Decatenation assays by topoisomerase II in the presence or absence of imatinib. Lane 1, K-DNA; lane 2, K-DNA with $1 \mathrm{U}$ of DNA topoisomerase II; lane 3, same as lane 2 plus $0.5 \mathrm{mM}$ etoposide; lane 4 , same as lane 2 plus $1 \mathrm{mM}$ Imatinib; lane 5, linear DNA marker; lane 6, decatenated DNA marker. 
to $18 \%, 14 \%$ and $7 \%$ upon serial dilutions (Fig. 4B). Residual nicked circular DNA present in the plasmid preparation was taken into account in the quantitative evaluations. We next employed the decatenation assays by topoisomerase II in the presence or absence of imatinib (Fig. 5). As seen in Fig. 5, the K-DNA was too large to enter the gel (Fig. 5, lane 1) whereas topoisomerase II yielded monomeric DNA rings of two species, OC-DNA and CCC-DNA (Fig. 5, lane 2). The reaction was completely abolished in the presence of etoposide (Fig. 5, lane 3). Likewise, $1 \mathrm{~mm}$ imatinib interfered with the decatenating activity of topoisomerase II (Fig. 5, lane 4) leaving catenated DNA molecules. The last two lanes indicate the linear (Fig. 5, lane 5) and catenated DNA (Fig. 5, lane, 6) markers.

Our results revealed that imatinib-induced apoptosis and inhibited cell proliferation in a dosedependent manner. Moreover, we also showed that the imatinib inhibited topoisomerase I and II enzymes activities in a dose-dependent manner, in vitro. The pharmacological significance of topoisomerases in anti-cancer drug development is wellknown (10-16). However, the compounds targeting these enzymes are structurally different, which suggests that there is not a unique mechanism for topoisomerase inhibition. Several studies in mammalian cells, employing cytotoxic agents with topoisomerase-targeting properties, suggest that the cellular responses were related to the formation of a drug/enzyme/DNA cleavable complex. Such a cleavable complex is formed, not through the inhibition of the catalytic activity of topoisomerases but through a mechanism involving the DNA damage (19). All known topoisomerase-targeting drugs, specifically and reversibly block the DNA rejoining, and results in the trapping of a covalent enzyme/ DNA intermediate $(26,27)$. The reversibility of the damage is a main difference between the actions of imatinib on topoisomerase reactions and apoptosis. Although our results showed that the imatinib promotes apoptosis through increasing caspase-3 enzyme activity and loss of mitochondrial membrane potential as well as interfering with the topoisomerase activities as shown in Figs 4 and 5, the concentrations used in two assays were different. As in the former test, an irreversible-damage is assessed, the concentrations of imatinib, expected to inhibit topoisomerase reactions and apoptosis, would not be expected to be the same.

\section{WHAT IS NEW AND CONCLUSION}

Unlike type I enzymes, identification of topoisomerase II-targeting chemicals is relatively recent. Our results demonstrate that imatinib blocked the decatenation activity of topo II, thereby suggesting a possible mechanism for imatinib-induced tumour cell-killing.

\section{ACKNOWLEDGEMENTS}

This study was partially supported by the grant from The Scientific and Technological Research Council of Turkey (Grant No: TBAG108T548) (Z.T.). We would like thank to Izmir Institute of Technology, Biotechnology and Bioengineering Research Facility staff for their technical help.

\section{REFERENCES}

1. Koca E, Haznedaroglu IC (2005) Imatinib mesylate and the management of chronic myeloid leukemia (CML). Turkish Journal of Hematology, 2, 161-172.

2. Baran Y, Ural AU, Gunduz U (2007) Mechanisms of cellular resistance to imatinib in human chronic myeloid leukemia cells. Hematology, 12, 497-503.

3. Rowley JD (1973) Letter: A new consistent chromosomal abnormality in chronic myelogenous leukaemia identified by quinacrine fluorescence and Giemsa staining. Nature, 243, 290-293.

4. Demetri GD, von Mehren M, Blanke CD et al. (2002) Efficacy and safety of imatinib mesylate in advanced gastrointestinal stromal tumors. The New England Journal of Medicine, 347, 472-480.

5. Schindler T, Bornmann W, Pellicena P, Miller WT, Clarkson B, Kuriyan J (2000) Structural mechanism for STI-571 inhibition of abelson tyrosine kinase. Science, 289, 1938-1942.

6. Druker BJ (2002) STI571 (Gleevec) as a paradigm for cancer therapy. Trends in Molecular Medicine, 8, 14-18.

7. Cassier PA, Dufresne A, Arifi S et al. (2008) Imatinib mesilate for the treatment of gastrointestinal stromal tumour. Expert Opinion on Pharmacotherapy, 9, 12111222.

8. Duffaud F, Le Cesne A (2009) Imatinib in the treatment of solid tumours. Targeted Oncology, 4, 4556.

9. Baran Y, Salas A, Senkal CE, Gunduz U, Bielawski J, Obeid LM, Ogretmen B (2007) Alterations of ceramide/sphingosine 1-phosphate rheostat involved in the regulation of resistance to imatinib-induced apoptosis in K562 human chronic myeloid leukemia 
cells. The Journal of Biological Chemistry, 282, 1092210934.

10. López-Lázaro M, Martín-Cordero C, Toro MV, Ayuso MJ (2002) Flavonoids as DNA topoisomerase I poisons. Journal of Enzyme Inhibition and Medicinal Chemistry, 17, 25-29.

11. Martín-Cordero C, López-Lázaro M, Gálvez M, Ayuso MJ (2003) Curcumin as a DNA topoisomerase II poison. Journal of Enzyme Inhibition and Medicinal Chemistry, 18, 505-509.

12. Chhatriwala H, Jafri N, Salgia R (2006) A review of topoisomerase inhibition in lung cancer. Cancer Biology and Therapy, 5, 1592-1599.

13. Ishar MPS, Singh G, Singh S, Sreenivasan KK, Singh G (2006) Design, synthesis, and evaluation of novel 6-chloro-/fluorochromone derivatives as potential topoisomerase inhibitor anticancer agents. Bioorganic and Medicinal Chemistry Letters, 16, 1366-1370.

14. Kucukoglu O, Ozturk B, Kamataki T, Topcu Z (2006) Inhibitory activities of Helichrysum taxa on mammalian type I DNA topoisomerase. Pharmaceutical Biology, 44, 189-193.

15. Alpan AS, Gunes HS, Topcu Z (2007) 1H-Benzimidazole derivatives as mammalian DNA topoisomerase I inhibitors. Acta Biochimica Polonica, 54, 561-565.

16. Topcu Z, Ozturk B, Kucukoglu O, Kilinc E (2008) Flavonoids in Helichrysum pamphylicum inhibit mammalian type I DNA topoisomerase. Zeitschrift für Naturforschung C, 63, 69-74.

17. Topcu Z, Borden KL (2000) The yeast two-hybrid system and its pharmaceutical significance. Pharmaceutical Research, 17, 1049-1055.

18. Wang JC (1996) DNA topoisomerases. Annual Review of Biochemistry, 65, 635-692.

19. Osheroff N (2001) Assay in DNA topoisomerase I relaxation activity. In: Stewart $\mathrm{L}$, Champoux JJ, eds. and DNA topoisomerase II-catalyzed DNA decatenation.
In: Haldane A, Sullivan DM, eds. DNA topoisomerase protocols, Part II: enzymology and drugs. Totowa, NJ: Humana Press, 1-23.

20. Topcu Z (2001) DNA topoisomerases as targets for anticancer drugs. Journal of Clinical Pharmacy and Therapeutic, 26, 405-416.

21. Baran Y, Gur B, Ural AU, Avcu F, Gunduz U (2007) Upregulation of multi drug resistance genes in doxorubicin resistant human acute myelogeneous leukemia cells and reversal of the resistance. Hematology, 12, 511-517.

22. Topcu Z, Castora FJ (1995) Mammalian mitochondrial DNA topoisomerase I preferentially relaxes supercoils in plasmids containing specific mitochondrial DNA sequences. Biochimica et Biophysica Acta, 1264, 377-387.

23. Sobhani AM, Ebrahimi S-A, Mahmoudian M (2002) An in vitro evaluation of human DNA topoisomerase I inhibition by Peganum harmala L. seeds extract and its beta-carboline alkaloids. Journal of Pharmacy and Pharmaceutical Sciences, 5, 19-23.

24. Topcu Z (2000) Densitometric quantification of DNA topoisomers in ethidium bromide-stained agarose gels and chemiluminescence-detected $X$ ray films. Acta Biochimica Polonical, 47, 835-839.

25. Marini JC, Miller KG, Englund PT (1980) Decatenation of kinetoplast DNA by topoisomerases. The Journal of Biological Chemistry, 255, 4976-4979.

26. Liu LF (1989) DNA topoisomerase poisons as antitumor drugs. Annual Review of Biochemistry, 58, 351375 (bu referans text içerisinde geçmiyor 25'ten $27^{\prime}$ ye geçilmiş).

27. Cozzarelli NR, Wang JC (1990) Anticancer drugs that convert DNA topoisomerases into DNA damaging agents. In: Liu LF, eds. DNA topology and its biological effects, New York, NY: Cold Spring Harbor Laboratory press, 371-389. 